\title{
A MOLECULAR LINE SURVEY OF THE CARBON STAR IRAS 15194-5115
}

\author{
L.- $\AA$. Nyman \\ ESO, Casilla 19001, Santiago 19, Chile \\ R.S. Booth, U. Carlström, L.E.B. Johansson, H.Olofsson \\ Onsala Space Observatory, S-439 00 Onsala, Sweden \\ R. Wolstencroft \\ Royal Observatory, Blackford Hill, Edinburgh EH9 3HJ, UK
}

IRAS 15194-5115 was discovered by IRAS and identified as a carbon star by Meadows et al. (1987). It is the third brightest carbon star at $12 \mu \mathrm{m}$, the brighter ones are IRC+10216 and CIT6. Its infrared properties are similar to those of IRC+10216. Le Bertre and Epchtein (1990) have monitored the star in the near-infrared and derived a period of 578 days. The distance to IRAS 15194-5115 is estimated to about $1 \mathrm{kpc}$ from infrared observations, and also by comparing its bolometric luminosity with that of IRC+10216.

We have used the 15m SEST (Swedish-ESO Submm Telescope) to make a survey of molecular lines toward IRAS 15194-5115 in the 3 and $1.3 \mathrm{~mm}$ bands. The idea was to derive molecular abundances and compare them with those of IRC+10216.

The observed lines are listed in Table 1, and a few spectra are shown in Fig. 1. In total 23 transitions of 14 molecular species and their isotopes have been detected. Abundances were calculated using the optically thin lines and the expression given by Olofsson et al. (1990). An excitation temperature of $10 \mathrm{~K}$ was assumed for all species, and their spatial extents were estimated from photodissociation calculations or in some cases scaled from interferometric observations of IRC+10216. The same species were also observed toward IRC+10216 with the SEST, and the abundances were calculated in the same way. The abundances for the two sources are similar to within a factor of 2 to 3 . From our CO data we estimate a mass loss rate of $5 \cdot 10^{-5}$ $\mathrm{M}_{\odot} \mathrm{yr}^{-1}$ and an expansion velocity of $22 \mathrm{~km} \mathrm{~s}^{-1}$ for IRAS 15194-5115.

The narrow, blueshifted component seen in the CO spectra in Fig. 1 emanates from an interstellar cloud in the line of sight. The CS(2-1) spectrum (Fig. 1f) shows a narrow, redshifted component whose intensity changes with time. It has a maximum near the infrared maximum. The intensity variations may be due to maser action.

\section{REFERENCES}

Le Bertre and Epchtein, 1990, "The infrared spectral region of stars", Montpellier Meadows et al., 1987, MNRAS 225, 43p

Olofsson et al., 1990, A\&A 230, 405 
TABLE 1. Observed lines, intensities, and abundances

\begin{tabular}{lclrl}
\hline Transition & $\begin{array}{c}\text { Frequency } \\
(\mathrm{MHz})\end{array}$ & $\begin{array}{l}\mathrm{T}_{\mathrm{mb}} \\
(\mathrm{K})\end{array}$ & $\begin{array}{c}\int_{\mathrm{T} b \mathrm{dv}} \\
\left(\mathrm{K} \mathrm{kms}^{-1}\right)\end{array}$ & $\begin{array}{l}\text { Abundance } \\
{[\mathrm{X}]\left[/ \mathrm{H}_{2}\right]}\end{array}$ \\
\hline $\mathrm{C}_{3} \mathrm{H}_{2}(2(1,2)-1(0,1))$ & 85338.9 & 0.02 & 0.95 & $1.1 \cdot 10^{-7}$ \\
$\mathrm{H}^{13} \mathrm{CN}(1-0)$ & 86340.2 & 0.57 & 20.4 & $7.4 \cdot 10^{-7}$ \\
$\mathrm{SiO}(2-1 v=0)$ & 86847.0 & 0.15 & 5.4 & $1.6 \cdot 10^{-7}$ \\
$\mathrm{HN}^{13} \mathrm{C}(1-0)$ & 87090.9 & 0.03 & 1.0 & $4.1 \cdot 10^{-8}$ \\
$\mathrm{C}_{2} \mathrm{H}(1-0)$ & 87316.9 & 0.12 & 12.5 & $9.9 \cdot 10^{-6}$ \\
$\mathrm{HCN}(1-0)$ & 88631.8 & 0.53 & 17.2 & Opt. thick \\
$\mathrm{HNC}(1-0)$ & 90663.5 & 0.09 & 3.4 & $9.6 \cdot 10^{-8}$ \\
$\mathrm{SiS}(5-4)$ & 90771.5 & 0.06 & 2.4 & $5.0 \cdot 10^{-7}$ \\
$\mathrm{HC} \mathrm{N}_{3}(10-9)$ & 90979.0 & 0.10 & 3.7 & $3.8 \cdot 10^{-7}$ \\
$\mathrm{C}_{4} \mathrm{H}(10-9)$ & 95150.3 & 0.04 & 3.5 & $1.4 \cdot 10^{-5}$ \\
$\mathrm{CS}(2-1)$ & 97981.0 & 0.43 & 16.4 & $1.1 \cdot 10^{-6}$ \\
$\mathrm{C}_{3} \mathrm{H}\left({ }^{2} \mathrm{M}_{3 / 2} \mathrm{~J}=9 / 2-7 / 2\right)$ & 97995.5 & 0.01 & 1.1 & $5.8 \cdot 10^{-8}$ \\
$\mathrm{C}_{3} \mathrm{~N}(11-10)$ & 108834.3 & 0.03 & 2.3 & $3.8 \cdot 10^{-7}$ \\
$\mathrm{SiS}(6-5)$ & 108924.3 & 0.06 & 2.3 & $3.8 \cdot 10^{-7}$ \\
$\mathrm{HC}{ }_{3} \mathrm{~N}(12-11)$ & 109173.6 & 0.07 & 3.1 & $3.9 \cdot 10^{-7}$ \\
$13 \mathrm{CO}(1-0)$ & 110201.4 & 0.35 & 15.3 & Opt. thick \\
$\mathrm{CN}(1-0)$ & 113491.0 & 0.15 & 16.4 & $1.1 \cdot 10^{-6}$ \\
$\mathrm{CO}(1-0)$ & 115271.2 & 1.3 & 49.0 & Opt. thick \\
$\mathrm{SiC}(5(0,5)-4(0,4))$ & 115383.0 & 0.08 & 2.8 & $3.5 \cdot 10^{-7}$ \\
$13 \mathrm{CO}(2-1)$ & 220398.7 & 0.78 & 32.0 & Opt. thick \\
$\mathrm{CO}(2-1)$ & 230538.0 & 3.5 & 121.5 & Opt. thick \\
$\mathrm{CS}(5-4)$ & 244935.6 & 0.57 & 18.4 & $3.5 \cdot 10^{-7}$ \\
$\mathrm{HCN}(3-2)$ & 265886.4 & 3.4 & 94.7 & Opt. thick \\
\hline & & & & \\
& & & &
\end{tabular}

1.0
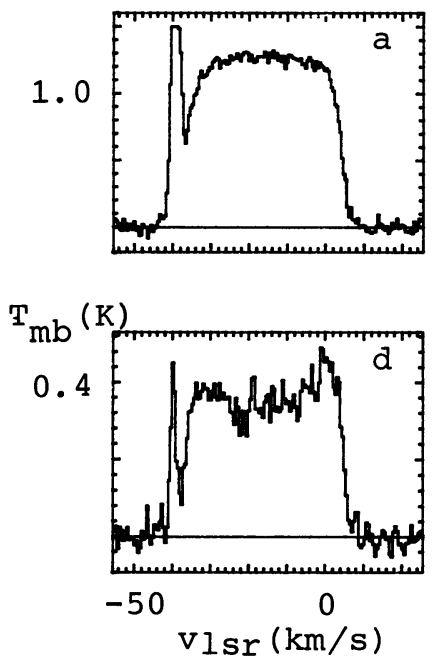
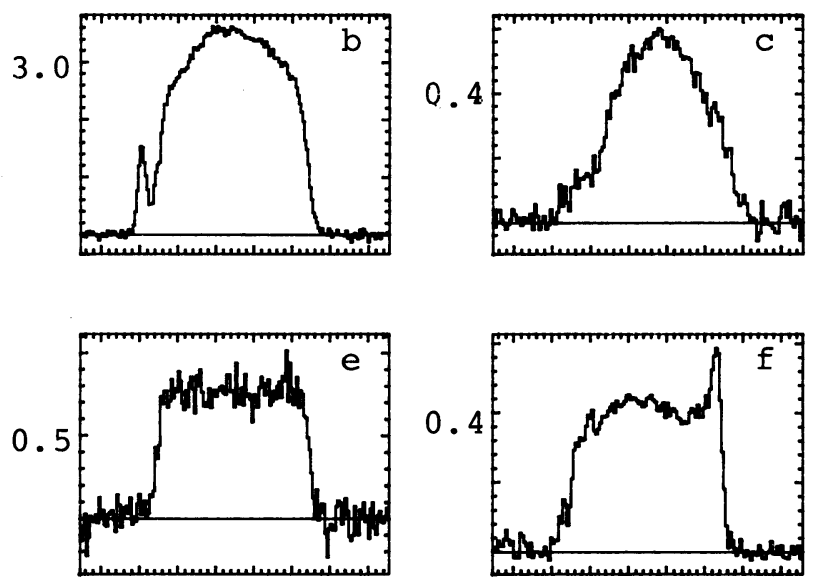

Figure 1a. Spectrum of $\mathrm{CO}(1-0)$, b. $\mathrm{CO}(2-1)$, c. $\mathrm{HCN}(1-0)$, d. ${ }^{13} \mathrm{CO}(1-0)$, e. ${ }^{13} \mathrm{CO}(2-1)$, f. $\mathrm{CS}(2-1)$. 